\title{
Evaluación del manejo avanzado de la vía aérea en un departamento de emergencia pediátrico
}

\section{Evaluation of advanced airway management in a pediatric emergency department}

\author{
Liz Nathalia Céspedes', Silvia Catalina González Vallejos', Laura Patricia Morilla', Sonia \\ Viviana Pavlicich ${ }^{1}$
}

\section{RESUMEN}

Introducción: La intubación endotraqueal en pacientes críticamente enfermos es un procedimiento de riesgo que requiere alta competencia en el manejo de la vía aérea. Es esencial conocer las habilidades de los médicos de emergencia pediátrica en el manejo avanzado de vías aéreas y de vías aéreas difíciles. Objetivo: Describir el manejo avanzado de vías aéreas y vías aéreas difíciles en un departamento de emergencia pediátrico. Materiales y Métodos: Estudio observacional, descriptivo y prospectivo en pacientes de 0 a 18 años que requirieron intubación endotraqueal en el periodo comprendido desde el 10 de mayo del 2018 al 31 de mayo del 2019. Se evaluó diagnóstico fisiopatológico, prevalencia de vía aérea difícil, aplicación de secuencia de intubación rápida, herramienta utilizada para la intubación, tiempo de intubación, intentos, operador y complicaciones. Se definió éxito al paciente intubado en dos intentos. Resultados: Se incluyeron 93 pacientes, la mediana de edad fue 10 (0.7192) meses . En 19 (20,4\%) pacientes se identificó predicción de vía aérea difícil, se realizó secuencia de intubación rápida en 91 (97,8\%) pacientes. El porcentaje de éxito fue $83,9 \%$ (78/93). En el primer intento 59,1\% (55/93) y en el segundo $50 \%$ (19/38). Se requirió cambio de operador en 19 oportunidades. Todos fueron intubados por laringoscopia convencional. La mediana para el tiempo de intubación fue 3 (2-5.5) minutos. La media del número de intentos fue 1,8 $( \pm 1,3)$. La complicación observada fue la desaturación de oxígeno en 23 (24,7\%) pacientes. Conclusión: La primera causa de intubación según el diagnóstico fisiopatológico fue la falla cardiopulmonar y la identificación de vía aérea difícil se presentó con relativa frecuencia.

Palabras clave: intubación endotraqueal, manejo avanzado de vía aérea, vía aérea difícil.

\begin{abstract}
Introduction: Endotracheal intubation in critically ill patients is a risky procedure that requires a high level of competence in airway management. It is essential to know the skills of pediatric emergency physicians in the advanced management of airways and difficult airways. Objective: To describe the advanced management of difficult airways and airways in a pediatric emergency department. Materials and Methods: This was an observational, descriptive and prospective study in patients from ages 0 to 18 years who required endotracheal intubation from May 10, 2018 to May 31, 2019. We evaluated pathophysiological diagnosis, prevalence of difficult airway, rapid intubation sequence intubation application, tools used for intubation, intubation time, attempts, operator characteristics and complications. Successful intubation patients was defined as intubation in two attempts. Results: 93 patients were included, the median of age was 10 (0.7-192) months. In 19 (20.4\%) patients, difficult airway prediction was identified, rapid intubation sequence was performed in $91(97.8 \%)$ patients. The percentage of success was $83.9 \%$ (78/93). In the first attempt 59.1\% (55/93) and in the second 50\% (19/38). Operator change was required 19 times. All were intubated by conventional laryngoscopy The median for intubation time was 3 (2-5.5) minutes. The average number of attempts was $1.8( \pm 1.3)$. The complication observed was oxygen desaturation in $23(24.7 \%)$ patients. Conclusion: The first cause of intubation according to the diagnosis pathophysiological was cardiopulmonary failure.Difficult airway identification was relatively frequent.
\end{abstract}

Keywords: Endotracheal intubation, advanced airway management, difficult airway.

\footnotetext{
${ }^{1}$ Hospital General Pediátrico Niños de Acosta Ñu. San Lorenzo, Paraguay.

Correspondencia: Silvia Catalina González Vallejos Correo:silgonval@gmail.com

Conflicto de interés: Los autores declaran no poseer conflicto de interés

Recibido: 08/08/2019 Aceptado: 8/11/2019

Doi: https://doi.org/10.31698/ped.46032019006
}

(cc) BY Este es un artículo publicado en acceso abierto bajo una Licencia Creative Commons CC-BY 4.0 


\section{INTRODUCCIÓN}

El manejo de la vía aérea es un aspecto importante de la atención de emergencia pediátrica. El acceso rápido y eficaz a las vías respiratorias puede significar la diferencia entre un buen resultado, la discapacidad o la muerte. El manejo óptimo requiere una comprensión de las diferencias entre niños y adultos con respecto a la anatomía y fisiología de las vías respiratorias, y la respuesta a los medicamentos ${ }^{(1)}$.

La intubación endotraqueal (IET) en pacientes críticos es un procedimiento de alto riesgo, que requiere una experiencia significativa en el manejo de la vía aérea y la comprensión de la fisiopatología del proceso de la enfermedad ${ }^{(2)}$.

Nuestro hospital es uno de los centros de referencia del país, con una afluencia importante de pacientes al servicio de emergencias, tanto de baja como de alta complejidad. Los médicos de guardia se enfrentan frecuentemente a pacientes con requerimiento de IET, por todo esto es necesario conocer las habilidades de los médicos de urgencia en el manejo avanzado de vías aéreas y vía aérea difícil (VAD); siendo esta última una condición que se define como la situación clínica en la que un anestesiólogo entrenado experimenta dificultad con la ventilación de la máscara facial de la vía aérea superior, dificultad con la intubación traqueal, o ambas ${ }^{(3)}$. Anticipar las dificultades en este procedimiento es esencial para obtener los resultados deseados.

El objetivo del presente trabajo fue describir el manejo avanzado de vías aéreas y vías aéreas difíciles en un departamento de emergencia pediátrico.

\section{MATERIALES Y MÉTODOS}

Estudio observacional, descriptivo y prospectivo con componente analítico, en el que se incluyeron por muestreo no probabilístico de casos consecutivos a todos los pacientes de 0 a 18 años que requirieron intubación endotraqueal a su ingreso al departamento de emergencia pediátrico (DEP) o encontrándose en observación en el mismo. Los datos fueron tomados por una persona asignada al momento del procedimiento exclusivamente para el llenado de un formulario tipo check list. Se excluyeron aquellos niños cuya ficha de recolección de datos no fue correctamente llenada.

Las variables estudiadas fueron: edad, sexo, peso, diagnóstico fisiopatológico, la predicción de VAD, uso de SIR (Secuencia de Intubación Rápida), herramienta utilizada (laringoscopio y video laringoscopio), tiempo total de intubación, número de intentos previos al éxito, el operador encargado del procedimiento, y las complicaciones inmediatas. El tiempo total de intubación se determinó desde el inicio de la laringoscopia hasta la fijación del tubo endotraqueal. El diagnóstico fisiopatológico se basó en el triángulo de evaluación pediátrica (TEP) con siete diagnósticos fisiopatológicos posibles (Estable, Disfunción del SNC, Dificultad respiratoria, Shock compensado, Falla respiratoria, Shock descompensado, Falla cardiorespiratoria) $)^{(4)}$. Se definió éxito al paciente intubado hasta en dos intentos.

Se registraron como complicaciones inmediatas: desaturación $<80 \%$, bradicardia para la edad y paro cardiorrespiratorio. Se definió VAD basado en la evaluación previa al procedimiento de: alteración en la evaluación externa (cara, cuello), grado de Mallampati, obstrucción de la vía aérea, incapacidad de movimiento cervical (collarín cervical en caso de traumatismo) $)^{(5)}$.

Los datos fueron analizados por SPSSV22. Las variables cuantitativas se expresan en medias con desvío estándar o medianas con rangos intercuartiles según su distribución. Las variables cualitativas se expresan en porcentajes. El estudio fue aprobado por el comité de investigación de la institución quien liberó el consentimiento informado.

\section{RESULTADOS}

En el periodo de estudio comprendido entre el 10 mayo del 2018 y el 31 de mayo del 2019 consultaron en el servicio de urgencias 113921 niños, demandaron internación 7031 pacientes de los cuales requirieron intubación endotraqueal (IET) 
120 pacientes, se analizaron 93 que cumplieron con los criterios de inclusión.
Los datos demográficos, el diagnóstico fisiopatológico y la predicción de VAD se describen en la Tabla 1.

Tabla 1. Datos demográficos, diagnóstico fisiopatológico, VAD*.

\begin{tabular}{lc}
\hline Variable & $\mathbf{N}=\mathbf{9 3}$ \\
\hline Edad (meses) & $10(0,7-192)$ \\
mediana (p25-75) & $7,5(2,4-58)$ \\
Peso (kg) & $\mathbf{N}(\mathbf{\%})$ \\
mediana (p25-75) & $36(38,7)$ \\
\hline Diagnostico Fisiopatológico & $40(43)$ \\
\hline Falla respiratoria & $14(15,1)$ \\
Falla cardiopulmonar & $3(3,2)$ \\
Disfunción del SNC & \\
Shock descompensado & $19(20,4)$ \\
VAD & $74(79,6)$ \\
Si & \\
No &
\end{tabular}

*VAD vía aérea difícil.

Los menores de 12 meses de edad fueron $62,3 \%$ (58/93) de los niños, de los cuales 50\% (29/58) tenían menos de 3 meses y $31 \%$ (9/29) fueron recién nacidos.

El operador del procedimiento de IET, el éxito, la necesidad de cambio de operador, la media de intentos de intubación, el éxito en la primera maniobra de intubación y el tiempo total para la IET, se describen en la Tabla 2.

Tabla 2. Características de la IET* en relación al operador, intentos y tiempo.

\begin{tabular}{lc}
\hline Variable & Paciente \\
\hline Operador & $\mathbf{N}(\%)$ \\
Médico emergentólogo & $49(52,7)$ \\
Fellow de emergencias & $38(40,8)$ \\
Médico pediatra & $4(4,3)$ \\
Intensivista & $1(1,1)$ \\
Anestesiólogo & $1(1,1)$ \\
Total & $\mathbf{9 3 ( 1 0 0 )}$ \\
\hline Éxito & \\
Si & $78(83,9)$ \\
No & $15(16,1)$ \\
Total & $\mathbf{9 3 ( 1 0 0 )}$ \\
\hline Cambio de operador & $\mathbf{1 9 ( 2 0 , 4 )}$ \\
\hline Éxito por operador/ número de intentos & \\
Médico emergentólogo & $53 / 72(73,6)$ \\
Médico pediatra & $3 / 4(75)$ \\
Fellow de emergencias & $29 / 44(65.9)$ \\
Anestesiólogo & $2 / 3(66,6)$ \\
Total de intentos & $\mathbf{1 2 3}(\mathbf{1 0 0})$ \\
\hline Intentos IET* & \\
media ( \pm DS) & $1,8 \pm 1,3$ \\
Tiempo de IET (min) & \\
mediana (p25-75) & $3(2-5,5)$ \\
\hline Inter
\end{tabular}

*IET intubación endotraqueal 
Se realizó SIR en 91 de los 93 pacientes, que representa el 97,8\%. La laringoscopía convencional fue aplicada en todos los pacientes. En el primer intento se intubaron 55/93 (59,1\%) y en el segundo 19/38 (50\%). Total de éxito (definido por intubación en hasta dos intentos) $78(83,9 \%)$.

La caída de la saturación de oxígeno fue la única complicación observada en el 24,7\% (23/93), siendo entre 79 y $70 \%$ en $13 / 93(13,9)$ y menor de $70 \%$ en
10/93 (10,75\%). Ningún paciente presentó bradicardia ni paro cardiorrespiratorio.

Entre los pacientes que fueron intubados en los dos primeros intentos (éxito) versus aquellos que lo hicieron en más de dos intentos existieron diferencias significativas en las complicaciones, observándose asociación con desaturación en los que requirieron más de dos intentos. Tabla 3.

Tabla 3. Comparación entre grupos con $\leq 2$ intentos vs $>2$ intentos IET*

\begin{tabular}{|c|c|c|c|}
\hline Variable & $\begin{array}{c}\text { Éxito ( } \leq 2 \text { intentos) } \\
\mathrm{n}(\%) \mathrm{n}=78\end{array}$ & $\begin{array}{c}\text { Fracaso ( }>2 \text { intentos) } \\
\mathbf{n}(\%) \mathrm{n}=15\end{array}$ & Valor $p$ \\
\hline \multicolumn{4}{|l|}{ Diagnóstico fisiopatológico } \\
\hline Falla respiratoria & $29(80,6)$ & $7(19,4)$ & ns \\
\hline Falla cardiopulmonar & $35(87,5)$ & $5(12,5)$ & \\
\hline Shock descompensado & $2(66,7)$ & $1(33,3)$ & \\
\hline Disfunción del SNC & $12(85,7)$ & $2(14,3)$ & \\
\hline VAD & $17(89,5)$ & $2(10,5)$ & ns \\
\hline \multicolumn{4}{|l|}{ Operador } \\
\hline Emergentólogo & $43(87,8)$ & $6(12,2)$ & ns \\
\hline Fellow de emergencias & $31(81,5)$ & $7(18,4)$ & \\
\hline \multicolumn{4}{|l|}{ Complicaciones } \\
\hline Sin complicaciones $(n=70)$ & $64(91,4)$ & $6(8,6)$ & $0,001 * *$ \\
\hline Saturación $<80 \%(\mathrm{n}=23)$ & $14(60,8)$ & $9(39,2)$ & \\
\hline Peso kg (mediana) & 7,7 & 5 & ns \\
\hline Edad meses (mediana) & 12 & 4 & $\mathrm{~ns}$ \\
\hline
\end{tabular}

* IET intubación endotraqueal; **test chi cuadrado.

\section{DISCUSIÓN}

En el presente estudio la primera causa de intubación según el diagnóstico fisiopatológico fue la falla cardiopulmonar. Se encontró que los niños con falla respiratoria requirieron con más frecuencia cambio de operador seguida de la falla cardiorespiratoria, aunque no se relacionó con el éxito en el procedimiento. Existen estudios que describen dificultad asociada a la condición fisiopatológica sean estas de origen respiratorio, cardiaco, hepático, renal o infeccioso; y según sea la causa se deberán de tomar las medidas correctivas ${ }^{(2)}$.

Más de la mitad de los pacientes tenían menos de un año, resultado que difiere de los hallazgos de Kerrey et al. en dos centros de Estados Unidos ${ }^{(6)}$. En nuestra casuística se podría explicar por la frecuencia de falla respiratoria en este rango etario que lleva a los niños pequeños a necesitar soporte ventilatorio, cabe resaltar que dentro del periodo del presente estudio se tuvo una epidemia respiratoria.

Se constató predicción de VAD en diecinueve pacientes, número superior a los hallazgos de Kerrey et al, donde solo el 3\% de los pacientes presentó $\mathrm{VAD}^{(6)}$. La alta prevalencia de VAD podría deberse a la presencia de niños con comorbilidades. La predicción de VAD no se asoció con mayor número de complicaciones ni con cambio de operador o aumento del número de intentos, esto podría deberse probablemente a la preparación 
previa; en nuestro centro una vez identificada la VAD el primer operador es el de mayor experiencia.

La SIR es el método de elección para la mayoría de las intubaciones pediátricas de emergencia y se asocia con una alta tasa de éxito y una baja tasa de eventos adversos graves ${ }^{(7)}$. Fue utilizada en casi la totalidad de los pacientes, con excepción de dos niños que se encontraban en paro cardiorrespiratorio al ingreso. Un resultado similar fue reportado en el estudio multicéntrico de Losek et al. ${ }^{(8)}$.

La totalidad de IET fueron realizados por laringoscopía directa. Este resultado está relacionado con que el equipo de videolaringoscopía es operado por los anestesiólogos en nuestra institución, quienes representan en el protocolo el respaldo ante la presencia de dificultad en la vía aérea y fueron excepcionalmente convocados durante el periodo de este estudio.

Más de la mitad de las intubaciones fueron realizadas en los primeros 3 minutos, en el primer o segundo intento, en su gran mayoría por el médico emergentólogo o el fellow de emergentología. En el hospital donde se realizó el estudio los residentes de pediatría no realizan intubaciones de emergencia, su entrenamiento se realiza con simuladores. Según un estudio realizado por Mulcaster y col. un profesional debe realizar 47 intubaciones para adquirir la destreza necesaria ${ }^{(9)}$.

Como es predecible el mayor porcentaje de éxito correspondió al médico emergentólogo, no pudiendo evaluarse competencias de pediatras por la baja frecuencia de la intervención, también descripto con anterioridad por Goton et al. ${ }^{(10)}$ Casi la totalidad de los pacientes fueron intubados en menos o igual a dos intentos, siendo necesario el cambio de operador en menos de la quinta parte de los pacientes. Es regla del servicio cambiar de operador sino se logra la intubación en dos intentos.

Este estudio aporta información sobre las habilidades de un equipo de profesionales en el manejo avanzado de la vía aérea que puede ser utilizada para introducir estrategias de mejoras.

\section{CONCLUSIÓN}

La primera causa de intubación según el diagnóstico fisiopatológico fue la falla cardiopulmonar y la identificación de vía aérea difícil se presentó con relativa frecuencia.

Más de la mitad de los pacientes tenían menos de un año de edad. Se utilizó la SIR en todos los pacientes que no acudieron en paro cardiorespiratorio.

\section{REFERENCIAS BIBLIOGRÁFICAS}

1. Sullivan K, Kissoon N. Securing the child's airway in the emergency department. Pediatric Emergency Care. 2002; 18(2):108-121.

2. Ahmed A, Azim A. Difficult tracheal intubation in critically ill. Journal of Intensive Care. 2018; 6:49. DOI: https://doi.org/10.1186/s40560-018-0318-4

3. Apfelbaum JL, Hagberg CA, Caplan RA, Blitt CD, Connis RT, Nickinovich DG, et al. Practice guidelines for management of the difficult airway: an updated report by the American Society of Anesthesiologists Task Force on Management of the Difficult Airway. Anesthesiology. 2013, 118(2):251-270. DOI: https://doi.org/10.1097/

\section{ALN.0b013e31827773b2}

4. Dieckmann RA, Brownstein D, Gausche-Hill M. The pediatric assessment triangle: a novel approach for the rapid evaluation of children. Pediatr Emerg Care. 2010; 26(4):312315. DOI: https://doi.org/10.1097/PEC.0b013e3181d6db37

5. Hagiwara Y, Watase H, Okamoto H, Tadahiro Goto, Hasegawa K. Prospective validation of the modified LEMON criteria to predict difficult intubation in the ED. Am J Emerg Med. 2015;33(10):1492-1496.

6. Kerrey BT, Rinderknecht AS, Geis GL, Nigrovic LE, Mittiga MR. Rapid sequence intubation for pediatric 
emergency patients: higher frequency of failed attempts and adverse effects found by video review. Ann Emerg Med. 2012; 60(3):251-259. DOI: https://doi.org/10.1016/j. annemergmed.2012.02.013

7. Sagarin MJ, Chiang V, Sakles JC, Barton ED, Wolfe RE, Vissers RJ, et al. Rapid sequence intubation for pediatric emergency airway management. Pediat Emerg Care. 2002; 18(6):417-423. DOI https://doi.org 10.1097/00006565200212000-00004

8. Losek JD, Olson LR, Dobson JV, Glaeser PW. Tracheal intubation practice and maintaining skill competency.
Pediatr Emerg Care. 2008; 24(5):294-299. DOI https://doi.org/10.1097/PEC.0b013e31816ecbd4

9. Mulcaster JT, Mills J, Hung OR, MacQuarrie K, Law JA, Pytka S, et al. Laryngoscopic Intubation: Learning and Performance. Anesthesiology, 2003; 98(1):23-27.

10. Goto T, Gibo K, Hagiwara Y, Okubo M, Brown DFM, Brown CA, et al. Factors Associated with First-Pass Success in Pediatric Intubation in the Emergency Department. West J Emerg Med. 2016; 17(1):129-134. DOI: https://doi.org/10.5811/westjem.2016.1.28685 